\title{
A Rare and Late Complication After Left Ventricular Assist Device Explantation
}

\author{
George Gradinariu, $\mathrm{MD}^{1 *}$, André R. Simon, MD, PhD², Jullien Gaer, MS, FRCS (C-Th) ${ }^{1}$ \\ ${ }^{1}$ Department of Cardiac Surgery, Harefield Hospital, Royal Brompton and Harefield NHS Foundation Trust, Harefield, Middlesex, \\ United Kingdom \\ ${ }^{2}$ Department of Cardiothoracic Transplantation and Mechanical Circulatory Support, Harefield Hospital, Royal Brompton and \\ Harefield NHS Foundation Trust, Harefield, Middlesex, United Kingdom
}

\begin{abstract}
Late complications can arise after explantation of a left ventricular assist device. We report the case of a patient who presented at the age of 19 years with peri-partum cardiomyopathy and was initially managed with a biventricular support device, which was subsequently upgraded to an ambulatory left ventricular assist device. This was successfully explanted after myocardial recovery via a minimally invasive approach 7 months later. The patient re-presented 5 years following explantation with hemoptysis. At redo sternotomy, a 10-cm remnant of the outflow graft was found to be eroding the surface of the right lung. The conduit was excised and the stump oversewn. Eleven months later, she presented again with hemoptysis, and a pseudo-aneurysm was identified on the computed tomography scan. She underwent urgent open repair with peripheral cannulation and deep hypothermic total circulatory arrest. The Dacron stump was found to be partially dehisced, leaving a $2-3 \mathrm{~cm}$ defect in the ascending aorta. The defect was repaired with a bovine pericardial patch. The patient made a good recovery and was successfully discharged.

Copyright (c) 2017 Science International Corp.
\end{abstract}

\section{Key Words:}

Ventricular assist device explantation - Outflow graft pseudo-aneurysm • Hemoptysis • Redo surgery

\section{Introduction}

Our center has previously reported successful explantation of left ventricular assist devices (LVADs) from patients who have been 'bridged-to-recovery' [1]. Here, we report a late complication of LVAD explantation in a woman who had been successfully bridged to recovery after developing a peri-partum cardiomyopathy.

\section{Case Presentation}

The patient first presented to our center in October 2009 at the age of 19 with peri-partum cardiomyopathy. She had severe globally impaired left ventricular function, with left ventricular ejection fraction (LVEF) estimated at $15 \%$. Supportive measures, including intra-aortic balloon pump, maximal anti-heart failure medication, and steroids proved inadequate. She remained ventilator-dependent and, approximately 2 months after her initial presentation, biventricular support was instituted with a Levitronix system $\left(\right.$ Levitronix $\left.{ }^{\circledast}\right)$. After another 2 months, the right-sided support was successfully weaned, and the left ventricular support was upgraded to an ambulatory HeartMate II device (Thoratec ${ }^{\circledR}$ ). One month later, the patient was successfully weaned from the ventilator and discharged home. In September 2010, her left ventricular function had recovered (LVEF $=67 \%)$, and

\footnotetext{
* Corresponding Author:

George Gradinariu, MD

Department of Cardiac Surgery

Harefield Hospital, Royal Brompton and Harefield NHS Foundation Trust

Hill End Road, Harefield, Middlesex, UB9 6JH, United Kingdom

Tel: +44 74876301 46; Fax: +44 1895822 870; E-Mail: george.gradinariu@nhs.net
} \\ Published by Science International Corp \\ ISSN 2325-4637 \\ tp://aorta.scienceinternational.org
}


she was admitted for explantation of the HeartMate device using the limited combined right anterior and left anterolateral thoracotomy approach previously described by our center [2]. The procedure was accomplished uneventfully, but due to dense adhesions, a length of the Dacron aortic outflow graft was not retrieved. The patient was discharged and continued cardiac rehabilitation with stable LVEF of $50-60 \%$ on follow-up echocardiography.

In January 2015, the patient presented to the emergency room with frank hemoptysis (estimated at around $400 \mathrm{~mL}$ ). Computed tomography (CT)-pulmonary angiography demonstrated an out-pouching $(2.1 \times 1.7 \mathrm{~cm})$ arising from the ascending aorta, which was surrounded by soft tissue density consistent with a pseudo-aneurysm (Figure 1A). Furthermore, a large opacity surrounded by ground glass changes was noted in the right upper pulmonary lobe. However, comparison of the mediastinal mass with previous images from a 2011 CT scan showed no significant changes in the appearance of the soft tissue around the ascending aorta (Figure 1B). Bronchoscopy showed no evidence of active bleeding from the right upper lobe, but a large amount of old blood was noted in the right lower lobe. The patient was taken to theater, and a $10-\mathrm{cm}$ length of Dacron tubing was identified as arising from the ascending aorta, representing the old outflow graft of the LVAD that was not retrieved when the device was explanted. Although there was no active bleeding from the graft, its tied-off distal end abutted against and had slowly eroded the right upper lobe surface, causing hemoptysis. The graft was mobilized and excised with oversewing of the stump arising from the

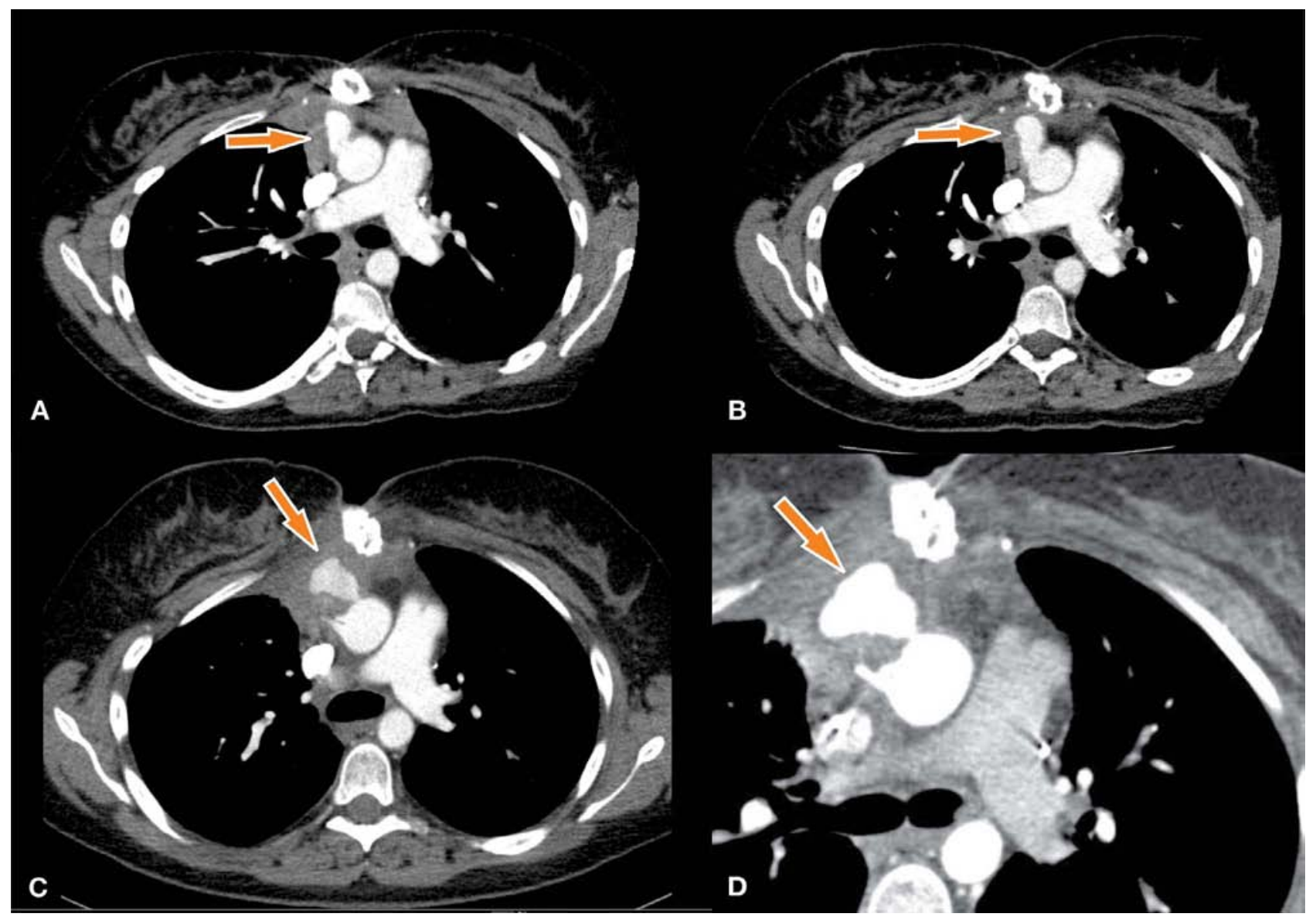

Figure 1. Panels $A$ and $B$. Computed tomography $(C T)$ scans showing the outflow graft stump one year after ventricular assist device explantation (Panel A) and before the redo sternotomy in January 2015 (Panel B). Panels C and D. CT scans in December 2015 showing the pseudo-aneurysm increase within two days. 
ascending aorta. A wedge of the chronically inflamed right upper lobe was also excised.

The patient was discharged and remained well on follow-up until July 2015, when a deep sternal wound infection became apparent. Sternal wires were removed, and a vacuum-assisted closure (VAC) dressing was inserted in November 2015. One week after the VAC dressing removal in December 2015, she again presented to the emergency room with recurrent hemoptysis. A CT scan showed a clear pseudo-aneurysm of the ascending aorta (Figure 1C). A repeat CT scan 2 days later showed the aneurysm to be enlarging (Figure 1D). The patient was therefore scheduled for urgent open repair. Cardiopulmonary bypass was instituted via right femoral venous and right axillary arterial cannulation. The apex of the left ventricle was vented via the old left anterior thoracotomy, and the patient was core-cooled to $18^{\circ} \mathrm{C}$. The sternum was divided and the pseudo-aneurysm entered. The dehiscence of the remaining graft stump was noted, which created a $2-3-\mathrm{cm}$ defect in the ascending aorta (Figure 2). During a 31min period of total circulatory arrest, the defect was repaired with a patch of bovine pericardium using pledgeted interrupted 3/0 Prolene mattress sutures. The patient recovered well but required temporary renal dialysis.

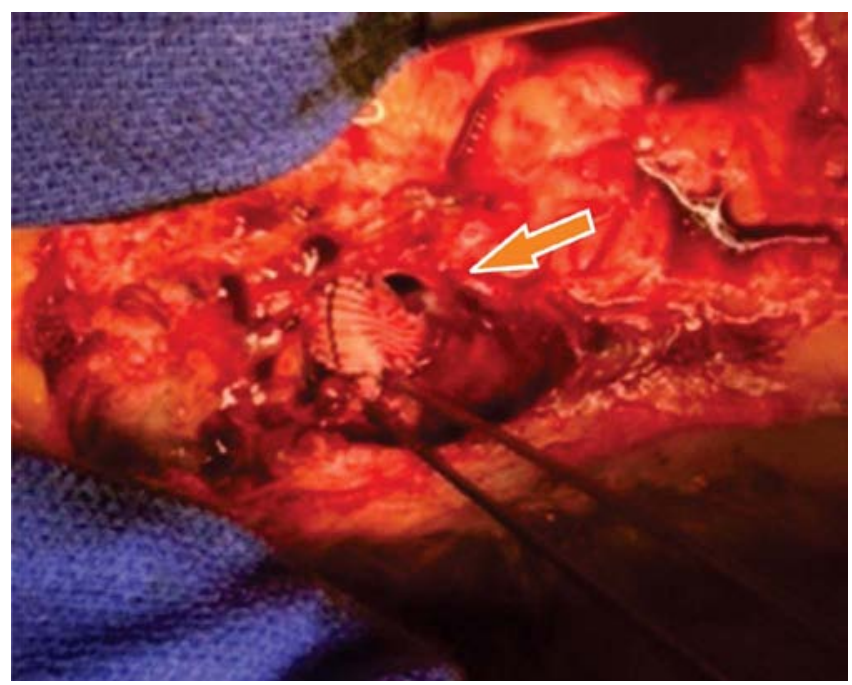

Figure 2. Intraoperative image showing the dehisced graft stump and defect in the ascending aorta.

\section{Discussion}

No clear consensus exists regarding the surgical approach for explanting ventricular assist devices. Although a minimally invasive approach should, at least in theory, be beneficial for the patient and the recovered myocardium, there is always a risk of leaving prosthetic material behind due to dense adhesions. The unusual presentation in this case was caused by an erosion of the lung surface caused by the residual stump of the outflow graft. Although previously reported after LVAD explantation, pseudo-aneurysms of the ascending aorta are rare, and the cause is usually infectious [3]. However, in this case, all intra-operative samples collected after repair of the pseudo-aneurysm were negative for bacteria or fungi. Thus, the cause of the dehiscence of the outflow graft stump and subsequent pseudo-aneurysm formation remains unclear.

Endovascular stenting has been reported as an option in similar cases [4] but requires custom-designed endografts. In our case, given the urgency and unsuitable anatomy, open repair was preferred. Furthermore, the relatively small size of the aortic defect and the absence of clinical features of infection mandated a simple patch repair as opposed to replacement of the entire ascending aorta. Thus, our clear preference in complex redo surgery for pseudo-aneurysms is peripheral arterial and venous cannulation with left ventricular apex venting through a limited left anterior thoracotomy and circulatory arrest during sternotomy and initial dissection of the heart.

\section{Conflict of Interest}

The authors have no conflicts of interest relevant to this publication.

Comment on this Article or Ask a Question 


\section{References}

1. Birks EJ, Tansley PD, Hardy J, George RS, Bowles $C T$, Burke $M$, et al. Left ventricular assist device and drug therapy for the reversal of heart failure. N Engl J Med. 2006;355:18731884. DOI: 10.1056/NEJMoa053063

2. Haj-Yahia S, Birks EJ, Dreyfus G, Khaghani A. Limited surgical approach for explanting the HeartMate II left ventricular assist device after myocardial recovery. J Thorac Cardiovasc Surg. 2008;135:453-454. DOI: 10.1016/j.jtcvs.2007.10.012

3. Kotschet E, Aggarwal A, Esmore D, Kaye
D. Left ventricular apical infection and rupture complicating left ventricular assist device explantation in 2 women with postpartum cardiomyopathy. J Heart Lung Transplant. 2005;24:350-354. DOI: 10.1016/j.healun.2003.12.005

4. Piffaretti $\mathrm{G}$, Cottini M, Carrafiello G, Castelli P, Beghi C, Mariscalco G. Endovascular repair of ascending aortic pseudoaneurysm with custom-designed endograft. Ann Thorac Surg. 2015;100:31-33. DOI: 10.1016/j.athoracsur.2015.02.118
Cite this article as: Gradinariu G, Simon AR, Gaer J. A Rare and Late Complication after Left Ventricular Assist Device Explantation. AORTA (Stamford). 2017;5(2):53-56. DOI: https://doi. org/10.12945/j.aorta.2017.16.032 